\title{
Return to Sport nach Handgelenkverletzungen
}

\author{
Ralf Oberhofer
}

\begin{abstract}
Nach einer Sportverletzung am Handgelenk ist ein frühzeitiges und gezieltes therapeutisches Handeln gefragt. Propriozeptive und sportspezifische Trainingsformen unterstützen den Rehabilitationsverlauf. Das Ziel ist eine erfolgreiche Rückkehr zum Sport bis hin zur Wettkampfbelastung. Erfahren Sie, wie ein progressiver Belastungsaufbau bei leichten und mittelschweren Verletzungen des Handgelenks aussehen kann.
\end{abstract}

Unmittelbar nach der Verletzung diagnostiziert zunächst ein Experte aus dem Fachbereich der Hand- oder Unfallchirurgie die genaue Strukturverletzung. Er entscheidet auch darüber, ob eine konservative oder operative Behandlung erfolgt, und stellt ein allgemeines Nachbehandlungsschema aus, an dem sich das weitere therapeutische Handeln orientiert ( $>$ Tab. 1). Im Folgenden prüft der Arzt in regelmäßigen Abständen den strukturellen Heilungsvorgang im Rahmen von verschiedenen klinischen und radiologischen Untersuchungen.

Aufgrund der vielfältigen Schadensbilder am Handgelenk kann kaum eine einheitliche Empfehlung über den konkreten Zeitpunkt der Wiederaufnahme der Belastung in einer Disziplin oder Sportart gegeben werden. Während schwere Handverletzungen im Fußball beispielsweise kaum Einfluss auf die Spielfähigkeit haben, können bereits kleine Blessuren am Handgelenk etwa beim Turnen, Gewichtheben, Kugelstoßen oder Stabhochsprung stark leistungsmindernd wirken. Ungefähr 10-20\% aller Sportverletzungen betreffen das Handgelenk und die Finger [2], wobei Knochen, Sehnen, Bänder und der trianguläre fibrokartilaginäre Komplex (TFCC) involviert sein können.

Das interdisziplinäre Team besteht in der Sportrehabilitation aus dem Arzt, dem Sportler, dem Trainer und dem/ den Therapeuten. Untereinander sind eine klare Kommunikation und Abstimmung unbedingt notwendig, um zielführend arbeiten zu können. Die Handtherapie startet nach der Immobilisations-/Schonungsphase.

Ziel der Rehabilitation Im Fokus sämtlicher Nachbehandlungs- und Rehabilitationsmaßnahmen steht neben der Wiederherstellung der ursprünglichen Leistungsfähigkeit und der Reduktion von Bewegungseinschränkungen und Gelenkinstabilitäten auch die Vermeidung von erneuten Verletzungen an derselben Stelle. Daher ist eine vorsichtige Rückführung in das belastende sportspezifische Training an- gezeigt, um das Auftreten von rezidiven Verletzungen zu verhindern. Wer zu schnell zu intensiv trainiert, riskiert seine Heilungschancen.

Auch verletzungsunabhängige und nichtmedizinische Faktoren wie sportliche, ökonomische, psychologische oder gar politische Aspekte können bei der Return-toSport-Entscheidung nach Handgelenk der Einheitlichkeit halber verletzungen im Einzelfall eine Rolle spielen. Der medizinische Aspekt sollte bei allen Erwägungen dennoch unbedingt im Fokus stehen, insbesondere um schwerwiegende Spätfolgen wie etwa eine Arthrose des Gelenks zu vermeiden.

Die therapeutische Rehabilitation beginnt mit der Befunderhebung und orientiert sich im Folgenden an den Wundheilungsphasen und an davon abgeleitete Rehabilitationsphasen. Der allgemeinen Handrehabilitation schließt sich die sportspezifische Rehabilitation an, in der der Sportler für seine individuelle Sportart vorbereitet wird.

\section{Die Handrehabilitation nach einer Sportverletzung}

Befunderhebung Zu Beginn erfasst der Therapeut die aktuellen Beschwerden des Sportlers in der Anamnese. Hinsichtlich des Schmerzes analysiert er nicht nur die Lokalisation und das 24-Stunden-Verhalten des Schmerzes, sondern auch die konkrete auslösende Bewegung.

Im Hinblick auf den Rehabilitationsverlauf und die weitere Trainingsgestaltung ist die Information über die Entstehung des Traumas von besonderer Bedeutung. So ist bei akuten Verletzungen durch einen Unfall eine fortschreitende Wundheilung zu erwarten. Chronische Verletzungen hingegen entstehen durch wiederholte Mikrotraumata als Folge ständiger Über- und Fehlbelastungen der 
- Tab. 1 Strukturverletzungen des Handgelenks mit allgemeinen ärztlichen Handlungsempfehlungen. Die dargestellten verschiedenen Therapiepfade basieren auf Erfahrungen aus der Normalbevölkerung [1].

\begin{tabular}{|c|c|c|c|c|}
\hline $\begin{array}{l}\text { Strukturverletzung } \\
\text { des Handgelenks }\end{array}$ & ärztliche Behandlung & $\begin{array}{l}\text { Beginn Physio-I } \\
\text { Handtherapie }\end{array}$ & $\begin{array}{l}\text { Return to Activity: Beginn } \\
\text { allgemeines Training }\end{array}$ & $\begin{array}{l}\text { Return to Sport: Beginn sportspezifi- } \\
\text { sches Training und Wettkampftrai- } \\
\text { ning bei handbelasteten Sportarten } \\
\text { (in Abhängigkeit von der Disziplin) }\end{array}$ \\
\hline Skaphoidfraktur & $\begin{array}{l}\text { konservativ: Gips } \\
\text { operativ: Schraubenos- } \\
\text { teosynthese }\end{array}$ & $\begin{array}{l}\text { 6.-12. Woche } \\
\text { funktionell }{ }^{*}\end{array}$ & $\begin{array}{l}\text { 2.-3. Woche mit immobili- } \\
\text { sierendem Gelenkschutz }\end{array}$ & ab 6. Monat \\
\hline \multirow{2}{*}{ distale Radiusfraktur } & konservativ: Gips & $\begin{array}{l}\text { 4.-6. Woche } \\
\text { funktionell }\end{array}$ & \multirow{2}{*}{$\begin{array}{l}\text { 2.-3. Woche mit immobili- } \\
\text { sierendem Gelenkschutz }\end{array}$} & \multirow{2}{*}{ ab 3.-4. Monat } \\
\hline & $\begin{array}{l}\text { operativ: Plattenosteo- } \\
\text { synthese }\end{array}$ & $\begin{array}{l}\text { 1.-2. Woche } \\
\text { bewegungsstabil * }\end{array}$ & & \\
\hline \multirow{2}{*}{ Tendovaginitis } & konservativ: Orthese & $\begin{array}{l}\text { 2.-3. Woche } \\
\text { funktionell }\end{array}$ & \multirow{2}{*}{$\begin{array}{l}\text { 2.-3. Woche Gelenkschutz } \\
\text { mit Bandage/Tape }\end{array}$} & \multirow{2}{*}{ ab 2.-3. Monat } \\
\hline & $\begin{array}{l}\text { operativ: Sehnenfach- } \\
\text { spaltung }\end{array}$ & $\begin{array}{l}\text { 1.-2. Woche } \\
\text { funktionell }\end{array}$ & & \\
\hline \multirow{2}{*}{ Verletzung des TFCC } & operativ: Arthroskopie & $\begin{array}{l}\text { 1.- } 2 \text {. Woche } \\
\text { funktionell }\end{array}$ & $\begin{array}{l}\text { 2.-3. Woche mit immobili- } \\
\text { sierendem Gelenkschutz }\end{array}$ & ab 3.-4. Monat \\
\hline & operativ: Naht & $\begin{array}{l}\text { 4.-6. Woche } \\
\text { funktionell }\end{array}$ & $\begin{array}{l}\text { 2.-3. Woche mit immobili- } \\
\text { sierendem Gelenkschutz }\end{array}$ & ab 4.-6. Monat \\
\hline $\begin{array}{l}\text { SL-Band-Instabilität/ } \\
\text { Teilruptur }\end{array}$ & $\begin{array}{l}\text { konservativ: Gips/ } \\
\text { Orthese }\end{array}$ & $\begin{array}{l}\text { 4.-6. Woche } \\
\text { funktionell }\end{array}$ & $\begin{array}{l}\text { 2.-3. Woche mit immobili- } \\
\text { sierendem Gelenkschutz }\end{array}$ & ab 3.-4. Monat \\
\hline SL-Band-Ruptur & $\begin{array}{l}\text { operativ: Naht/ } \\
\text { Kirschnerdraht }\end{array}$ & 8. Woche & $\begin{array}{l}\text { 2.-3. Woche mit immobili- } \\
\text { sierendem Gelenkschutz }\end{array}$ & ab 4.-6. Monat \\
\hline
\end{tabular}

Hand. Dies kann zu Instabilität und degenerativer Veränderung bis hin zur Stressfraktur, etwa des Skaphoids, führen. Die konservative Behandlung erfordert dann eine mehrere Monate andauernde Belastungsreduktion; nicht selten ist auch eine Operation notwendig. Die Selbsteinschätzung des Sportlers über die funktionelle Belastbarkeit seiner Hand kann Hinweise für eine gute oder schlechte Prognose hinsichtlich des Return to Sport geben.

Der Inspektion und Palpation der Gelenk- und Weichteilstrukturen folgt die aktive und passive Funktionsuntersuchung inklusive Stabilitätstests des Handgelenks. Von besonderer Bedeutung ist die Widerstandsprüfung aller extrinsischen und intrinsischen Muskeln auf Kraft und Belastungsfähigkeit. Bei einer neurologischen Symptomatik wie etwa einem Taubheitsgefühl in den Fingern erfolgt zudem eine neurologische Untersuchung.

\section{Rehabilitationsprinzipien}

Das Ziel des Rehabilitationsprogramms ist die Schaffung einer Umgebung, in der die verschiedenen an der Wundheilung relatierten Prozesse stattfinden können und alle inhibierenden negativen Faktoren eliminiert werden ([3]).

- Tab. 2 zeigt einen 5-Phasen-Plan der Sportrehabilitation.
Die therapeutischen Maßnahmen orientieren sich an der Physiologie des Bindegewebes, des Knorpels sowie der Physiologie der Frakturheilung bei knöchernen Verletzungen.

Die Wundheilung des Bindegewebes kann in drei Phasen eingeteilt werden:

1. Die akute Entzündungsphase dauert 2-5 Tage. In dieser Phase giltes den Abbau von nekrotischem Gewebe und den Vorgang der Blutstillung und -gerinnung zu unterstützen.

2. In der von Tag 6-21 andauernden Proliferationsphase werden Kollagenfasern vom Typ III gebildet. Eine gute Durchblutung des Gewebes verbessert die Neubildung von Kollagen und Matrixkomponenten. Diese wird ab der initialen Rehabilitationsphase mit schmerzadaptierten assistierten/aktiven Bewegungen erzielt.

3. In der Remodelierungsphase werden die Typ-III-Kollagene langsam durch progressive Strukturbelastung in Kollagenfasern vom Typ I umgewandelt, welche nach 300-500 Tagen die vollständige Belastungsfähigkeit erreichen. Diese Adaptionen können durch spezifische Strukturbelastung und insbesondere durch ein gesteigertes sportspezifisches und propriozeptives Training optimiert werden.

Das Knorpelgewebe benötigt zur Ernährung intermittierende Belastungswechsel und aktive Mobilisation, darüber hi- 
- Tab. 2 Rehabilitationsprinzipien nach Sportverletzungen [1].

\begin{tabular}{|c|c|c|c|c|}
\hline $\begin{array}{l}\text { Wundhei- } \\
\text { lungsphase }\end{array}$ & Rehabilitationsphase & Pathologie & Behandlungsziele & Rehamaßnahmen \\
\hline $\begin{array}{l}\text { Entzündungs- } \\
\text { phase }\end{array}$ & akute Verletzung & $\begin{array}{l}\text { Gewebeverletzung (Hämatom, } \\
\text { Ödem, Inflammation, Nekrose) }\end{array}$ & $\begin{array}{l}\text { Schutz, Reduktion der } \\
\text { Verletzung, Schwellung und } \\
\text { Schmerzen }\end{array}$ & $\begin{array}{l}\text { Erstversorgung nach PECH-Regel (Pause, } \\
\text { Eis, Kompression, Hochlegen) }\end{array}$ \\
\hline \multirow{2}{*}{$\begin{array}{l}\text { Proliferations- } \\
\text { phase }\end{array}$} & $\begin{array}{l}\text { initiale Rehabilitation } \\
\text { (Return to Activity) }\end{array}$ & $\begin{array}{l}\text { Fibroblastenstadium, regrediente } \\
\text { Entzündung und Ödem } \\
\text { Zugfestigkeit (0-15\%) }\end{array}$ & $\begin{array}{l}\text { progressive schmerzfreie } \\
\text { Range of Motion (ROM) }\end{array}$ & $\begin{array}{l}\text { aktiv-assistive Bewegung, kurzzeitiger } \\
\text { Widerstand, leichte Isometrie, lockeres } \\
\text { Aufwärmtraining }\end{array}$ \\
\hline & $\begin{array}{l}\text { progressive } \\
\text { Rehabilitation }\end{array}$ & $\begin{array}{l}\text { frühe Gewebereparation, einfaches } \\
\text { Kollagen und Gewebereifung } \\
\text { Zugfestigkeit (15-50\%) }\end{array}$ & $\begin{array}{l}\text { Steigerung der ROM, } \\
\text { Steigerung der Kraft, } \\
\text { limitierte Übungen, Schutz }\end{array}$ & $\begin{array}{l}\text { passive und aktive Bewegung, Dehnen, } \\
\text { zunehmender Widerstand, isokinetische } \\
\text { Übungen, forciertes Aufwärmtraining }\end{array}$ \\
\hline \multirow{2}{*}{$\begin{array}{l}\text { Remodelie- } \\
\text { rungsphase }\end{array}$} & integrierte Funktion & $\begin{array}{l}\text { reifes Kollagen, klare Gewebe- } \\
\text { strukturen, Zugfestigkeit } \\
(50-90 \%)\end{array}$ & $\begin{array}{l}\text { gesteigerte Übungen und } \\
\text { Kraft, aufgehobener Schutz }\end{array}$ & $\begin{array}{l}\text { gesteigerte Übungen gegen Widerstand, } \\
\text { Dehnungsübungen, koordinatives und } \\
\text { propriozeptives Training }\end{array}$ \\
\hline & Return to Sport & $\begin{array}{l}\text { Geweberemodellierung, reife } \\
\text { Gewebestrukturen Zugfestigkeit } \\
(90-99 \%)\end{array}$ & $\begin{array}{l}\text { maximale Übungen, } \\
\text { Integration, Vorbeugung }\end{array}$ & $\begin{array}{l}\text { Dehnung und Erhaltung der Kraft, } \\
\text { gesteigertes koordinatives Training, } \\
\text { Vorbeugung der erneuten Verletzung }\end{array}$ \\
\hline
\end{tabular}

naus eine gute funktionelle Belastbarkeit zum Schutz des subchondralen Knochens. Haarrisse in diesem Bereich benötigen ca. 4-6 Wochen Belastungsreduktion zur Ausheilung.

\section{Propriozeptives Training in der initialen, progressiven und integrierten Rehabilitationsphase}

Das propriozeptive Training zur Reinnervationsschulung sollte bereits in der initialen Rehabilitationsphase beginnen, um eine gute Entwicklung der Mobilität, Koordination und funktionellen Stabilität des Handgelenks zu gewährleisten. Einfache Faustschluss- und Fingerextensionsübungen mit leichten Widerständen sind Basisübungen für Patienten/ Sportler in der Frühphase der Handrehabilitation. Hierzu können sie kleine Gel- und Softbälle, elastische Handtrainer oder einstellbare Greifklammern bei der Ausführung verwenden. Das Training sollten sie mehrfach täglich für ca. 5 Minuten mit kleinen Pausen und angepasstem Widerstand eigenständig schmerzadaptiert durchführen.

Propriozeptive Neuromuskuläre Fazilitation Eine hervorragende therapeutische Technik bei Handgelenkverletzungen ist die Propriozeptive Neuromuskuläre Fazilitation (PNF). Ziel dabei ist es, die spezielle Neurophysiologie der Strukturen, Funktionseinheiten und -ketten der Aktivitäten zu rehabilitieren. Zu den Grundprinzipien der Technik zählen dreidimensionale Bewegungsmuster, die in der sporttechnischen Analyse von Bewegungsabläufen wiedererkannt werden können. An der oberen Extremität unterscheiden wir zwei Körperdiagonalen: Die Diagonale 1 verläuft über das Schultergelenk aus der Flexion, Abduktion, Außenrotation in die Extension, Adduktion, Innenrotation und umgekehrt. Die Diagonale 2 verläuft aus der Flexion, Adduktion, Außenrotation in die Extension, Abduktion, Innenrotation und umgekehrt.
Bei der Einleitung der Bewegungsimpulse ist die Hand sowohl bei der Stützmotorik als auch bei der Zielmotorik von distal nach proximal das erste Glied. Während „Stützmotorik" einen Übungskomplex im geschlossenen System beschreibt (Stützen mit den Händen gegen einen stabilen Angriffspunkt wie den Boden), bezeichnet „Zielmotorik“ Bewegungen in der offenen Kette, z. B. Wurfbewegungen, PNF-Diagonale). Der Therapeut arbeitet mit speziellen Grifftechniken, die zielgerichtet auf Gelenk- und Weichteilstrukturen in der Gliederkette Hand-Ellbogen-Schulter einwirken. Zu den propriozeptiven Stimuli zählt neben den Dehnreizen und Traktions-Kompressions-Reizen insbesondere auch der Bewegungswiderstand.

Schlüsselpunkt für die Bahnung von Funktionsbewegungen bildet der sog. optimale Widerstand, den der Therapeut dem Sportler/Patienten vorgibt. Zu Beginn der Proliferationsphase kann im Einzelfall auch eine assistive Unterstützung der Bewegung notwendig sein. Der Therapeut arbeitet mit taktilen, visuellen und akustischen Reizen, also mit der Außenwahrnehmung (Exterozeption) des Patienten, um die Motorik zu bahnen. Bewegungen in Extensionsrichtung starten mit der extensorischen Handkomponente und dem Kommando „Finger/Hand öffnen“, bei Bewegungen in Flexionsrichtung lautet das Kommando „Finger/Hand schließen“. Der Patient hält die Anspannung der Muskulatur für 7-10 s, bevor der Therapeut das Kommando „Muskelspannung lösen“ gibt. > Abb. 1 zeigt eine PNF-Technik mit rhythmischer Stabilisation zum Aufbau des Muskeltonus am Handgelenk in der Mittelstellung einer Diagonalen.

Grundspannung und Gelenkzentrierung Grundlegendes Ziel des propriozeptiven Trainings ist, neben der optimalen Ansteuerung der Muskeln im richtigen Zeitpro- 


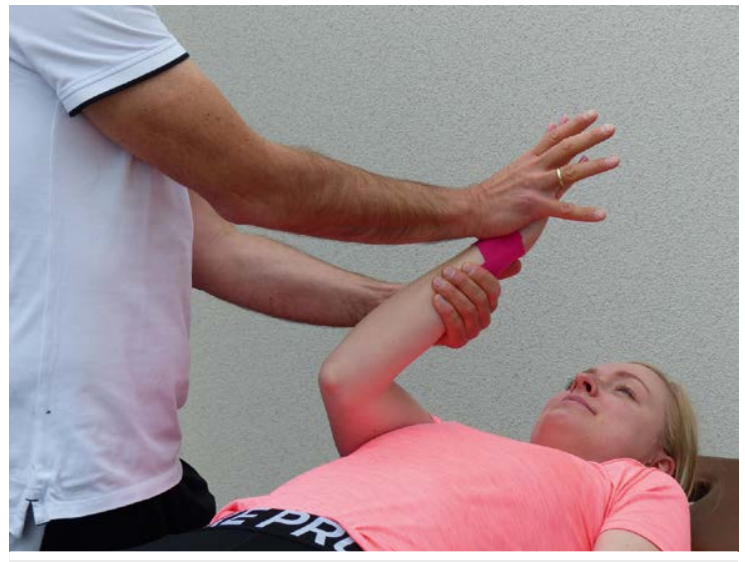

- Abb. 1 PNF-Diagonale 2 (Hand- und Fingerstreckung): Der Therapeut gibt einen propriozeptiven Traktionsimpuls sowie einen Bewegungswiderstand entgegen der zu stabilisierenden Bewegungsbahn. (Quelle: @ R. Oberhofer)

gramm auch eine Tiefenstabilität im Gelenk zu erhalten (siehe Kasten Hintergrundwissen). Ausgehend von dem „Stabilisierenden System “ nach Panjabi [4], welches aus einem aktiven und passiven System sowie einem Kontrollsystem besteht, geht es bei der Behandlung von strukturellen und funktionellen Instabilitäten somit um zwei Dinge:

- den Aufbau eines muskulären Haltetonus zum Schutz der passiven Kollagenstrukturen

- das richtige Timing bei zentrierter axialer und physiologischer Belastung des Handgelenks

\section{HINTERGRUNDWISSEN}

Ein Zeitprogramm beschreibt den Kurvenverlauf, der Geschwindigkeit, Dauer und Höhe des Kraftanstieges in der Muskulatur während einer bestimmten Bewegung aufzeigt. Am Ende der Rehabilitation soll der Sportler in seinen sportspezifischen Bewegungsmustern wieder das schnelle Zeitprogramm mit hohen Kraftspitzen trainieren können. Der Aufbau des propriozeptiven Trainings erfolgt in der initialen Rehabilitationsphase mit einem anderen Zeitprogramm (isometrische Grundspannung) und niedrigeren Belastungsintensitäten.

Eine besondere Bedeutung kommt dabei den lokalen gelenkzentrierenden Muskeln zu: Anders als beispielsweise die Schulter besteht der Muskelapparat am Handgelenk nicht lokal aus oberflächigen und aus tiefen Muskeln. Ein extrinsischer Muskelkomplex führt vom Ellbogen und Unterarm über den Karpus und inseriert an den Metakarpalbasen; ein anderer Teil führt zu den Mittel- und Endphalangen. Vom Karpus und Metakarpus ausgehend verläuft ein intrinsischer Muskelkomplex, der zu den Phalangen führt und die Fingerbewegungen koordiniert und stabili- siert. Eine Kokontraktion von Handgelenksflexoren und -extensoren unter Einbindung der Metakarpal-Stabilisatoren (Mm. lumbricales und interossei) führt zu einer guten Gelenkzentrierung des Handgelenks. Die abduzierenden Muskeln von Daumen und Kleinfinger unterstützen dabei die Grundspannung der Hohlhand. Diese basiert auf Flexion/Abduktion der MCP-Gelenke und einer leichten Flexion (ca. $10^{\circ}$ ) in PIP- und DIP-Gelenken sowie ca. $30^{\circ}$ Dorsalextension im Handgelenk.

Übung zum Aufbau der Grundspannung und der Gelenkzentrierung:

- Der Patient richtet Finger und Handgelenk achsengerecht aus: Bei ausgestreckten Fingern konvergieren die Endglieder der Finger zum Os capitatum, bei flektiertem PIP- und DIP-Gelenk zum Os scaphoideum [5]. Der Unterarm wird in der Nullstellung positioniert, anschließend auch in der Pro- und Supination.

- Nun gibt der Therapeut am Handgelenk des Patienten/Sportlers einen Traktions- und Gleitimpuls nach palmar, also in die instabile Richtung. Der Impuls ist nur so stark, dass die Gelenkrezeptoren stimuliert werden und der Patient wahrnehmen kann, in welche Richtung die Anspannung erfolgen muss ( $\triangleright$ Abb. 2 ).

- Anschließend erzeugt der Patient/Sportler aktiv eine kräftige, gelenkzentrierende Muskelspannung am Handgelenk. Der Gelenkkontakt zwischen Handwurzel und Unterarmknochen wird dadurch gesteigert, der Patient zieht die Handwurzelknochen sozusagen aktiv an den Unterarm heran.

Neben dieser therapeutisch begleiteten Übung kann der Patient/Sportler mit der Ballhalteposition eine Erhöhung der Stiffness (Stabilität) des Handgelenks erreichen ( $\mathbf{A b b}$. 3). Dadurch erfolgt die Einbindung der Mm. abductor pollicis longus, extensor carpi radialis longus, flexor carpi ulnaris und pronator quadratus durch Kokontraktion.

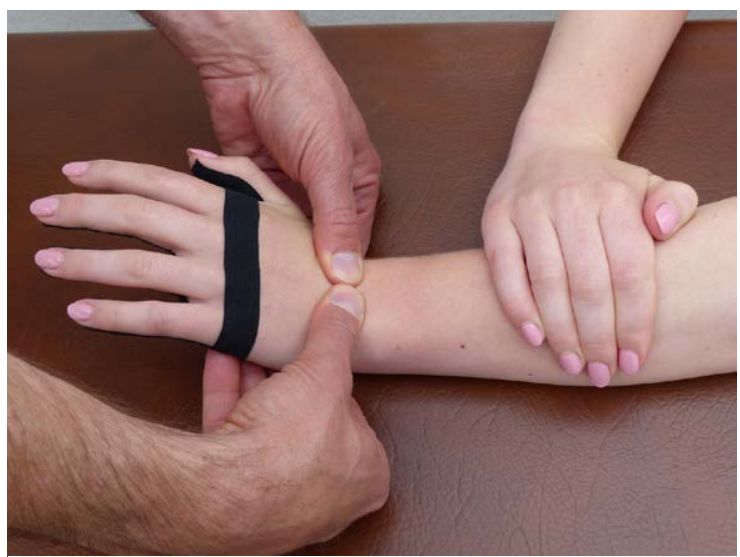

Abb. 2 Therapeutische Technik bei struktureller und funktioneller Instabilität des Handgelenks: Auf den passiven Impuls des Therapeuten in Richtung der Instabilität spannt der Patient seine Muskulatur aktiv in Richtung der stabilen Position an. (Quelle: @ R. Oberhofer) 


\section{VORSICHT}

Bei einer SL-Band-Instabilität ist mit der Dart-Throwing-Motion des Handgelenks zu starten, um möglichst wenig Stress auf die verletzte Struktur auszuüben. Diese Handgelenkbewegung verläuft - wie beim Werfen eines Dartpfeils - von dorsalradial nach palmar-ulnar und zurück.

Belastungssteigerung Grundsätzlich erfolgen die propriozeptiven Übungen zuerst statisch (Grundspannung), dann unter dynamischer Kontrolle auch langsam konzentrisch/exzentrisch und letztendlich reaktiv (konzentrisch/ exzentrisch maximal schnell). Zudem kann die Belastung mit Hebelverlängerungen in Form von distalen Widerständen und Lasten, dem erhöhten Einsatz des Körpergewichtes oder über Geschwindigkeitssteigerung von Bewegungen (z. B. beim Armschwung oder bei Wurf- und Stoßbewegungen) gesteigert werden. Wichtig ist dabei, dass der Patient die Stabilität der Hand auch während der Pro- und Supination im Unterarm, der Flexion und Extension im Ellbogen sowie bei sämtlichen Schulterbewegungen auch mit der höheren Last halten kann.
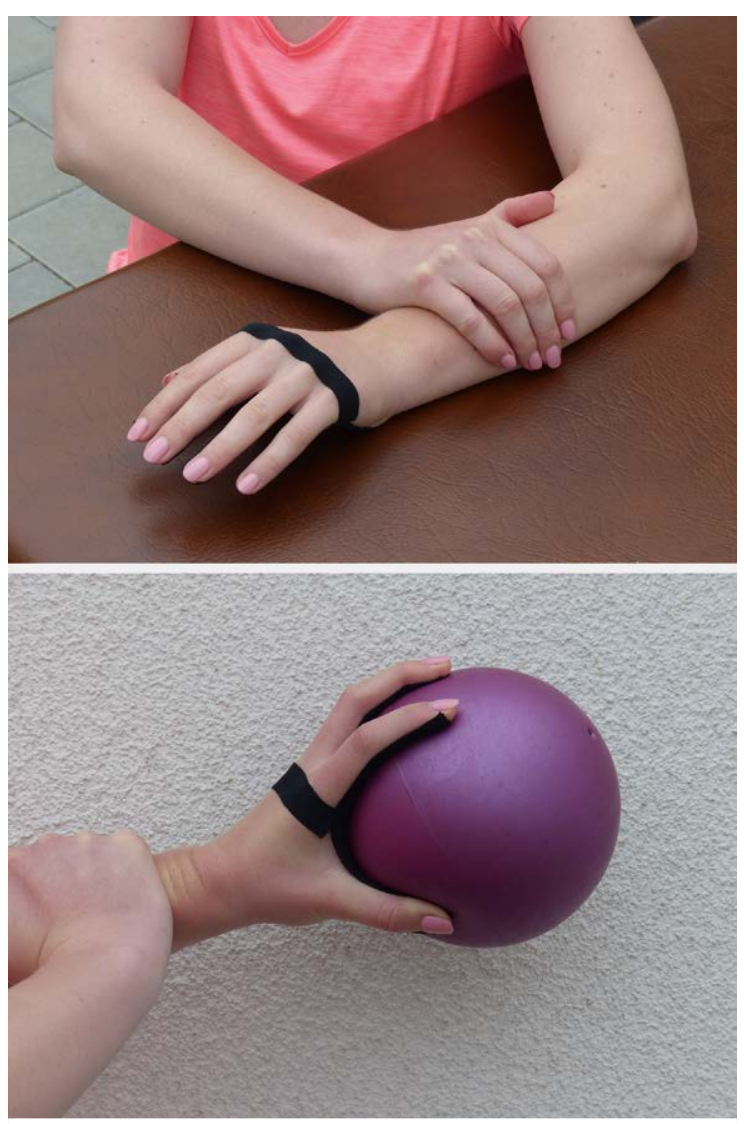

- Abb. 3 Eigenübung „Ballhalteposition“: a Aufbau der Grundspannung ohne Ball. b Übung mit Ball. (Quelle: (c) R. Oberhofer)
Mit einem leichten Gymnastikball (ca. 300 Gramm) können diese Bewegungen bis hin zur Wurf- und Stoßbewegung zunächst langsam als Imitationsübung eingeleitet werden. Es folgt ein fließender Übergang zu den sportspezifischen Bewegungsmustern. Das sensomotorische propriozeptive Training wird dann auch ohne vollständige visuelle Kontrolle schnellkräftigend mit reaktiver Belastung der oberen Extremität ausgeführt. Durch die schnelle Ansteuerung der Muskulatur wird die dynamische Stabilität des Handgelenks auch im Hinblick auf die Verletzungsprophylaxe verbessert.

Krafterhaltung In den angrenzenden Gelenken ist ab der initialen Rehaphase ein gesteigertes Widerstandstraining erforderlich, um stärkere Atrophien in der oberen Extremität zu verhindern. Dafür wird das Lastmoment proximal der Verletzung angebracht. In Unterarmstützposition kann beispielsweise mit dem Sling-Trainer die komplette Rumpfmuskulatur trainiert werden. Der Sling-Trainer eignet sich darüber hinaus auch hervorragend zum Training der Greif-, Halte- und Stützfunktion der Hände, insbesondere durch Gewichtsverlagerungen des Körperschwerpunkts ( A Abb.4).

Return to Activity Parallel zur Physio-/Ergotherapie steigt der Sportler meist in das allgemeine Grundlagentraining seiner Sportart ein, wobei das verletzte Handgelenk unbedingt mit einem Gelenkschutz zu stabilisieren ist. Der Trainer sollte also aktiv in den Rehabilitationsprozess seines Athleten einbezogen werden. Im Fokus stehen dabei zunächst die „On-Field-Grundlagen“ Ausdauer, Kraft, Schnelligkeit, Beweglichkeit und Koordination. Häufig kann der Sportler bereits sehr schnell wieder am Lauf-, Sprung- und Athletiktraining teilnehmen, da dabei das Handgelenk nicht oder nur wenig beansprucht wird.

\section{Sportspezifische Rehabilitation}

Das Anforderungsprofil der konditionellen und technischkoordinativen Fähigkeiten einer Sportdisziplin ist die Grundlage für ein zielgerichtetes allgemeines und spezifisches Aufbautraining. Ein Sportphysiotherapeut profitiert dabei von eigenen Erfahrungswerten und guten Trainingskenntnissen der Kernsportarten, um weiterführend und in Abstimmung mit dem Trainer erfolgreich die integrierte Rehabilitationsphase zu gestalten.

Technisch-koordinatives Training Für viele Sportarten zählt das sog. Lauf-, Sprung- und Wurf-ABC zum allgemeinen technischen koordinativen Training. Damit gemeint sind Grundübungen zur Entwicklung der Koordination in den elementaren Bewegungen dieser Bereiche.

Sportler mit Handgelenkverletzung beginnen koordinative Lauf- und Sprung-Übungen mit einem Gelenkschutz. Aufgrund des schnellkräftigen Armschwunges benötigen sie allerdings auch hier eine stabile Grundspannung in den Händen und müssen daher unbedingt auf eine korrekte 


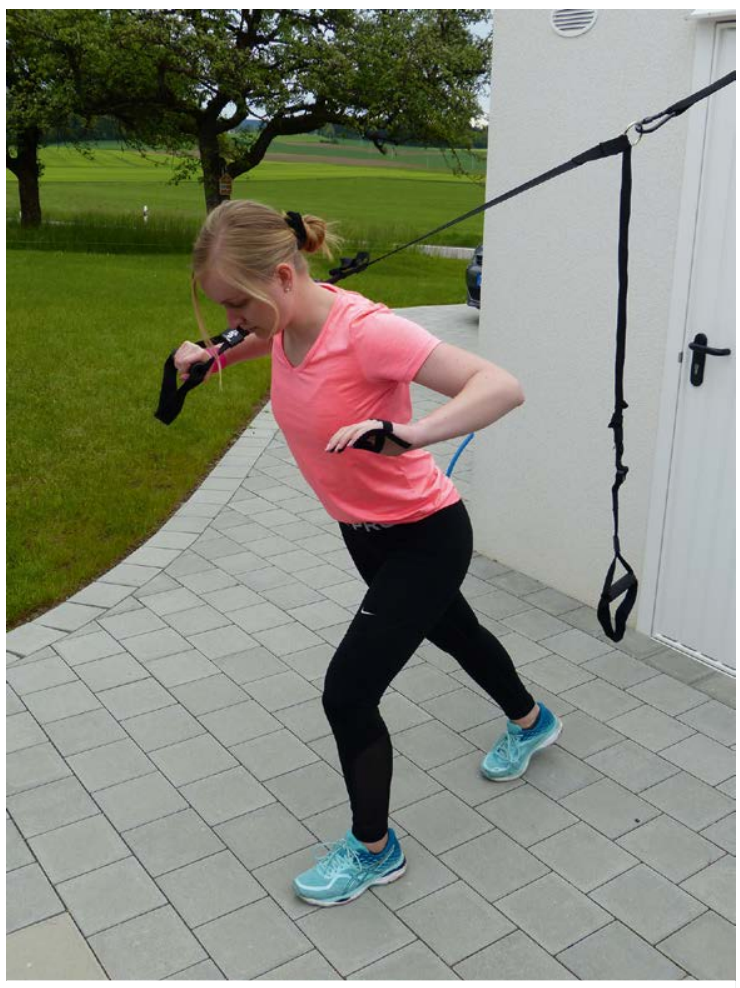

- Abb. 4 Einarmige Übung am Sling-Trainer mit Gewichtsverlagerung zur Verbesserung der Stützfunktion: Das Schultergelenk ist $90^{\circ}$ abduziert. Schulter, Ellbogen und Handgelenk befinden sich in einer Ebene unterhalb der Horizontalen. Durch Gewichtsverlagerung auf den Haltegriff des Sling-Trainers wird die Stützkraft des gesamten Armes trainiert. (Quelle: @ R. Oberhofer)

Ausführung achten. Zu den Übungen zählen das Skipping ( = schnelle Sprinterbewegung), der Kniehebelauf, der Hopserlauf, der Sprunglauf oder andere kleine Sprünge.

Werfen und Stoßen hingegen erfordert richtige „Handarbeit", die nicht nur Stärke, sondern auch Feingefühl in den Händen voraussetzt: Der Werfer muss das Wurfgerät zum einen in eine günstige Halteposition bringen, um es abwerfen zu können. Zum anderen erfolgt die Energieübertragung von den Beinen über den Rumpf, weiter über die Arme und schließlich in die Hand, sodass das Gerät mit höchster Energie in Wurfrichtung abgeworfen werden kann. Auf eine technisch korrekte Armführung mit richtiger Kraftübertragung über die Hände ist dabei zu achten. Mit einem Medizinball mit unterschiedlichen Gewichten $(0,8-3 \mathrm{~kg}) \mathrm{kann}$ diese spezifische Koordination auch in therapeutische Einheiten integriert werden. Zu den Übungsreihen des Wurf-ABC zählen ein- und beidarmige Würfe und Stöße in verschiedenen Variationen ( $\triangleright$ Abb. 5). Bei den Ballsportarten sollte auch der jeweilige Ball als Trainingsgerät in der Sporttherapie eingesetzt werden. Bei Kontaktsportarten wie Handball gilt es auch den Einsatz der Hände im Angriff- und Abwehrverhalten zu trainieren ( A Abb. 6).

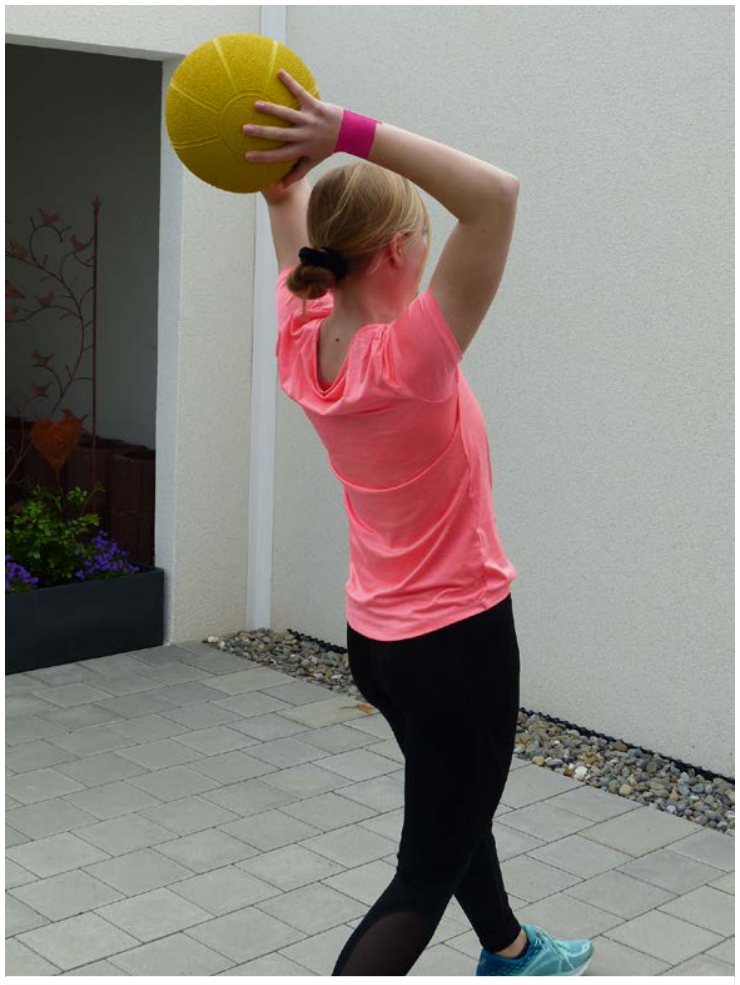

Abb. 5 Das beidarmige Werfen eines Medizinballs gegen eine Wand ist eine Übung aus dem Wurf-ABC. (Quelle: (c) R. Oberhofer)

Beim Turnen zählen neben Übungen des Wurf-ABC insbesondere der Handstand, das Rad am Boden, der Aufschwung und Stütz am Reck und das Schwingen und Kehren am Barren zu den elementaren Bewegungstechniken. Hier ist eine Handgelenksstütze für den distalen Unterarm und den Karpus zur Verletzungsprophylaxe besonders wichtig.

Allgemeines Krafttraining Zur allgemeinen Vorbereitung für die obere Extremität bietet sich ein progressives Krafttraining an Geräten an. Besonders empfehlenswerte Übungen sind Rudern, Brustpresse, Latissimus-Zug, Bizeps-/TrizepsCurls und Übungen am Zugapparat für die Innen- und AuBenrotation der Schulter. Wichtig ist, bei der Ausführung auf die Handstabilität zu achten. Diese Übungen können für 2-3 Wochen jeweils mit 3-5 Sätzen und 15-20 Wiederholungen begonnen werden. Anschließend kann die Wiederholungszahl zuerst auf 10-15 und dann auf 5-8 Wiederholungen reduziert werden, wobei progressiv dazu die Gewichte erhöht werden. Um die Anpassungsreaktionen in Form einer Muskelhypertrophie bei ca. 8-15 Wiederholungen oder der Maximalkraftsteigerung bei 1-8 Wiederholungen zu erreichen, muss eine schmerzfreie muskuläre Ausbelastung erfolgen. Das gesamte Training erfolgt unter Einhaltung von Regenerationszeiten 2-3x pro Woche.

Ein allgemeines Stabilisationstraining des Handgelenks gehört in der Sportvorbereitung zur täglichen Hausaufgabe 


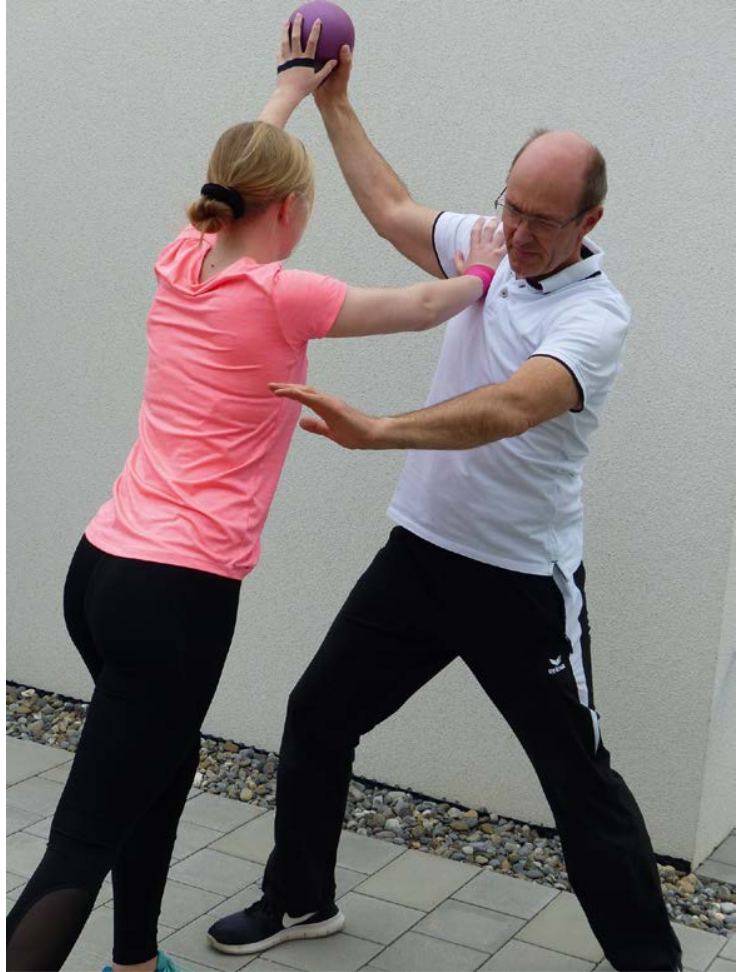

Abb. 6 Angriffs-/Abwehrverhalten: Halten und Schieben des Abwehrspielers beim Handball. (Quelle: $\odot$ R. Oberhofer)

des Sportlers. Er kann dazu spezielle Handtrainer wie Greifklammern oder -ringe, Schwingstäbe, Gelbälle oder das Powerweb nutzen. Auch diese Übungen werden bis zur schmerzfreien Ausbelastung durchgeführt.

Spezifisches Krafttraining Dazu gehört bei vielen Kraftund Schnellkraftdisziplinen, insbesondere in der Leichtathletik, das Training mit der Langhantel. Dieses setzt eine vollständige Belastungsfähigkeit des Handgelenks voraus, um Übungen wie das Bankdrücken, Bankziehen, Umsetzen oder Reißen leistungssteigernd und mit höchsten Lasten auszuführen. In der progressiven Rehabilitationsphase können daher nur einzelne Technikelemente des Langhanteltrainings vorbereitend trainiert werden.

Return to Sport/Return to Competition Der Wiedereinstieg in den Sport bzw. Wettkampf hängt aus medizinischer Sicht vom Schweregrad des Traumas, der vollständigen knöchernen Konsolidierung, der Zugfestigkeit des kollagenen Bindegewebes, der Stabilität des TCCC und von der Belastung des Handgelenks in der jeweiligen Sportdisziplin ab. Er kann parallel zur Sportreha verlaufen oder sich an diese anschließen. Erst nach Wiederaufnahme des sportspezifischen Trainings (Return to Sport) kann der Sportler bzw. sein Trainer einen genauen Zeitpunkt für den ersten Wettkampf/das erste Spiel unter Berücksichtigung des Therapiefortschritts bestimmen (Return to Competition).

\section{Fazit}

Für optimale Therapieerfolge sind eine gute Abstimmung und Kommunikation in einem interdisziplinären Team bedeutend. Darüber hinaus sollte der Sportler fehlerhafte Trainingsmethoden selbstkritisch reflektieren können und bereit sein, Anweisungen von Therapeuten und Ärzten anzunehmen. Der sportliche Wettkampf erfordert nicht nur eine vollständige Belastungsfunktion der Hand, sondern auch eine gute konditionelle und technisch-koordinative Leistungsfähigkeit. Zusätzlich muss der Sportler mental bereit dazu sein, die Hand wieder uneingeschränkt zu belasten. In handbetonten Sportarten sollte er insbesondere nach dem Return to Competition eine Handgelenksstützbandage zur Verletzungsprophylaxe tragen. Dies führt auch dazu, dass sich der Sportler sicherer fühlt und sich die Belastung besser zutraut.

\section{Autorinnen/Autoren}

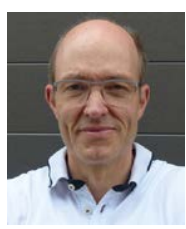

\section{Ralf Oberhofer}

Physiotherapeut, Sportphysiotherapeut I.A.S., Manualtherapeut, C-Trainer in der Sportart Leichtathletik. Er ist selbst ehemaliger Leistungssportler in der Leichtathletik im Zehnkampf und arbeitet im Arbeitsplatzspezifischen Rehabilitationszentrum (ASR) mit Schwerpunkt Nachbehandlung von Arbeits- und Sportunfällen in Weingarten als fachliche Leitung der Physiotherapie.

\section{Korrespondenzadresse}

\author{
ASR Weingarten \\ Gaußstraße 3 \\ 88250 Weingarten \\ Deutschland
}

Literatur

[1] Honigmann P, Häfeli M, Lardi A et al. Return to Sports nach typischen Handverletzungen. Schweizer Zeitschr Sportmed und Sporttraumatol 2012; 60: 60-69

[2] Henke T. Gesundheitsbericht NRW, Ministerium für Gesundheit, Soziales, Frauen und Familie des Landes NRW, Bielefeld; 2003

[3] Van Wingerden BAM. Bindegewebe in der Rehabilitation. Schaan/Liechtenstein: Scipro; 1998

[4] Van den Berg F. Stabilisierendes System Panjabi. In: Van den Berg F, Hrsg. Angewandte Physiologie 1: Das Bindegewebe des Bewegungsapparates verstehen und beeinflussen. Stuttgart: Thieme; 2007

[5] Hirt B, Seyhan H, Wagner M, Zumhasch R. Anatomie und Biomechanik der Hand. Stuttgart: Thieme; 2012 